\title{
Relating multihadron production in hadronic and nuclear collisions
}

\author{
Edward K.G. Sarkisyan ${ }^{1,2, a}$, Alexander S. Sakharov ${ }^{3,4, b}$ \\ ${ }^{1}$ Department of Physics, The University of Texas at Arlington, Arlington, TX 76019, USA \\ ${ }^{2}$ Department of Physics, CERN, 1211 Geneva 23, Switzerland \\ ${ }^{3}$ Department of Physics, Wayne State University, Detroit, MI 48202, USA \\ ${ }^{4}$ TH Division, Department of Physics, CERN, 1211 Geneva 23, Switzerland
}

Received: 21 May 2010 / Revised: 19 September 2010 / Published online: 11 November 2010

(C) The Author(s) 2010. This article is published with open access at Springerlink.com

\begin{abstract}
The energy-dependence of charged particle mean multiplicity and pseudorapidity density at midrapidity measured in nucleus-nucleus and (anti)proton-proton collisions are studied in the entire available energy range. The study is performed using a model, which considers the multiparticle production process according to the dissipating energy of the participants and their types, namely a combination of the constituent quark picture together with Landau relativistic hydrodynamics. The model reveals interrelations between the variables under study measured in nucleusnucleus and nucleon-nucleon collisions. Measurements in nuclear reactions are shown to be well reproduced by the measurements in $\mathrm{pp} / \overline{\mathrm{p}} \mathrm{p}$ interactions and the corresponding fits are presented. Different observations in other types of collisions are discussed in the framework of the proposed model. Predictions are made for measurements at the forthcoming LHC energies.
\end{abstract}

1. Soft hadron multiparticle production is one of the most intriguing topics in high-energy interaction studies. Data have been investigated in different types of interactions, ranging from lepton-lepton to nucleus-nucleus interactions, and over a large energy span, covering several orders of magnitude. QCD, the theory of strong interactions, has provided partonic description of many observations. However, the problem of soft multiparticle production still eludes a complete understanding and remains one of the challenging problems in high-energy physics [1]. The new high-energy data from LHC provide an opportunity to look at the system under new conditions. Of special interest are nucleusnucleus collisions, probing nuclear matter at extreme conditions, where new forms of matter are expected to be created

\footnotetext{
a e-mail: sedward@mail.cern.ch

b e-mail: Alexandre.Sakharov@cern.ch
}

at very high densities and temperatures. The data available from RHIC experiments allow an interesting comparison of the particle production mechanisms with the less complex $\mathrm{e}^{+} \mathrm{e}^{-}$and pp systems. In this context, the global, or bulk, variables such as the average charged particle multiplicity and particle densities (spectra), which are the first available experimental observables, are of fundamental interest [1-3] as they are sensitive to the underlying interaction dynamics.

In this paper we consider the center-of-mass (c.m.) energy dependence of the average multiplicity and near midrapidity density of charged hadrons produced in nucleusnucleus and (anti)proton-proton collisions. Whereas the multiplicity is sensitive mostly to the fraction of energy being transformed into observed particles in a given reaction, the midrapidity density reflects different stages of the reaction. Both variables increase with the collision c.m. energy. Recent measurements at RHIC follow the trends observed in $\mathrm{e}^{+} \mathrm{e}^{-}$and proton-proton interactions. The values of both bulk variables are found $[4,5]$ to be similar when comparing the measurements in $\mathrm{e}^{+} \mathrm{e}^{-}$interactions at the c.m. energy of $\sqrt{s_{\mathrm{ee}}}$, and in most central ("head-on") heavy-ion collisions at the nucleon-nucleon c.m. energy $\sqrt{s_{\mathrm{NN}}}=\sqrt{s_{\mathrm{ee}}}$, where the measurements in the latter case are normalized to the number of pairs of participants ("wounded" nucleons [6, 7]). This phenomenon is found to be independent of the type of colliding nucleus for $\sqrt{s_{\mathrm{NN}}}$ between $\sim 20$ and $200 \mathrm{GeV}$.

Assuming a universal mechanism of hadron production is present in both types of interaction, and that it is driven only by the amount of energy involved into secondary production, one would expect the same value of the observables to be obtained in proton-proton collisions when $\sqrt{s_{\mathrm{pp}}}$ is almost equal to $\sqrt{s_{\mathrm{NN}}}$. However, comparing these data $[8$, 9] to the measurements from RHIC, one finds [4, 5, 10-18] significantly lower values in hadron-hadron collisions. Furthermore, the recent RHIC data from deuteron-gold interactions at $\sqrt{s_{\mathrm{NN}}}=200 \mathrm{GeV}$ unambiguously points to the same 
values of the mean multiplicity as measured in antiprotonproton collisions $[5,19]$.

To interpret these findings, we have proposed in [20] a phenomenological description based on the energy dissipation by colliding participants into the state formed during the early stage of the collision. Particle production is then driven by the amount of the initial effective energy deposited in this early phase by the relevant types of participants. The experimental observations referred to above have been shown to be well described by this model and further predictions have been made. In this paper, the new and higher-energy data are added and analyzed.

2. In our consideration, the whole process of a collision is treated as the expansion and the subsequent break-up into particles from an initial state, in which the total available energy is assumed to be concentrated in a small Lorentzcontracted volume. There are no restrictions due to the conservation of quantum numbers other than energy and momentum constraints, thus allowing a relation between the amount of energy deposited in the collision zone and the features of bulk variables in different reactions. This approach resembles the Landau phenomenological hydrodynamical description of multiparticle production in relativistic particle collisions [21, 22]. Though the hydrodynamical description does not match ideally the data on multiparticle production in the whole range of pseudorapidity and different particle species, it gives good agreement with the multiplicity measurements in such different reactions as nucleusnucleus, $\mathrm{pp}, \mathrm{e}^{+} \mathrm{e}^{-}$and $\nu \mathrm{p}$ collisions demonstrating striking predictive power [5, 17, 23-29, 31, 32]. Recently, the Landau model prediction for the Gaussian pseudorapidity shape due to the longitudinal particle transport has been shown to reproduce well the RHIC data $[18,33]$ as well as $[31,32]$ the phenomenon known as the "limiting fragmentation" [34]. The latter was demonstrated to be independent of the energy and types of colliding objects [5, 25-29]. This indicates that the main assertions of the Landau approach are useful to estimate fractions of the energy dissipated into particles produced in different reactions, particularly in nucleus-nucleus collisions [35]. Let us stress here that in this paper the Landau hydrodynamical model is considered in the frame of the constituent quark picture as it is described below.

Once the collision of the two Lorentz-contracted particles has resulted in a fully thermalized system, but before expansion, we assume that the production of secondary particles is defined by the fraction of energy of the participants deposited in the volume of thermalized system at the moment of collision. This implies that there is a difference between results of collisions of structureless and composite particles: in composite particle collisions not all the constituents deposit their energy when they form a small
Lorentz-contracted volume of the thermalized initial state. Therefore, in nucleon-nucleon collisions the interactions occur between single constituent, or dressed, quarks in accordance with the additive quark picture [36, 37], and the other quarks are considered to be spectators. Thus the energy of the initial thermalized state which is responsible for the number of produced secondary particles is that of the interacting single quark pair. The quark spectators which are not part of the thermalized volume at the moment of collision do not participate in secondary particle production. As a result, the leading particles $[38,39]$ resulting from the spectator quarks carry away a significant part of the energy. Thus, only about $1 / 3$ of the entire nucleon energy is available for particle production in $\mathrm{pp} / \overline{\mathrm{p}} \mathrm{p}$ collisions.

In heavy ion collisions, however, more than one quark per nucleon interacts due to the large size of the nucleus and to the long travel path inside the nucleus. The more central the nucleus-nucleus collision is, the more interactions occur and the larger is the energy available for secondary particle production. In central nuclear collisions, a contribution of constituent quarks rather than participating nucleons seems to determine particle production and their distributions [40]. In the most central collisions, the density of matter is so high (almost saturated) that all three constituent quarks from each nucleon may participate nearly simultaneously in the collision, depositing their energy coherently into the thermalized collision volume. In this case, the entire energy of the participating nucleons is available for bulk production in headon nucleus-nucleus collisions. Comparing this to protonproton collisions, where only one out of three constituent quarks from each proton interacts, one expects the features of the bulk variables per pair of participants measured in the most central heavy-ion interactions to be similar to those from proton-proton collisions but at a three times larger c.m. energy, $\sqrt{s_{\mathrm{pp}}} \simeq 3 \sqrt{s_{\mathrm{NN}}}$.

Adding together the above discussed ingredients, namely the Landau model and the constituent quark picture, one finds for the ratio of the charged particle rapidity density $\rho(y)=\left(2 / N_{\text {part }}\right) d N_{\mathrm{ch}} / d y$ per participant pair at the midrapidity value $y=0$ in heavy-ion reaction, $\rho(0)$, to the density $\rho_{\mathrm{pp}}(0)$ in $\mathrm{pp} / \overline{\mathrm{p}} \mathrm{p}$ interaction,

$\frac{\rho(0)}{\rho_{\mathrm{pp}}(0)}=\frac{2 N_{\mathrm{ch}}}{N_{\mathrm{part}} N_{\mathrm{ch}}^{\mathrm{pp}}} \sqrt{\frac{L_{\mathrm{pp}}}{L_{\mathrm{NN}}}}$.

Here, $N_{\text {part }}$ is the number of participants $\left(N_{\text {part }}=2\right.$ in nucleon-nucleon interactions), $N_{\mathrm{ch}}$ and $N_{\mathrm{ch}}^{\mathrm{pp}}$ are the mean multiplicities in nucleus-nucleus and $\mathrm{pp} / \mathrm{p} \mathrm{p}$ interactions, respectively, and $L=\ln \frac{\sqrt{s}}{2 m}$ with $m$ being the mass of a participant, e.g. $m=m_{\mathrm{p}}$, the mass of the proton, in nucleusnucleus collisions. According to our model, we compute the ratio (1) for the rapidity density $\rho(0)$ and the multiplicity 


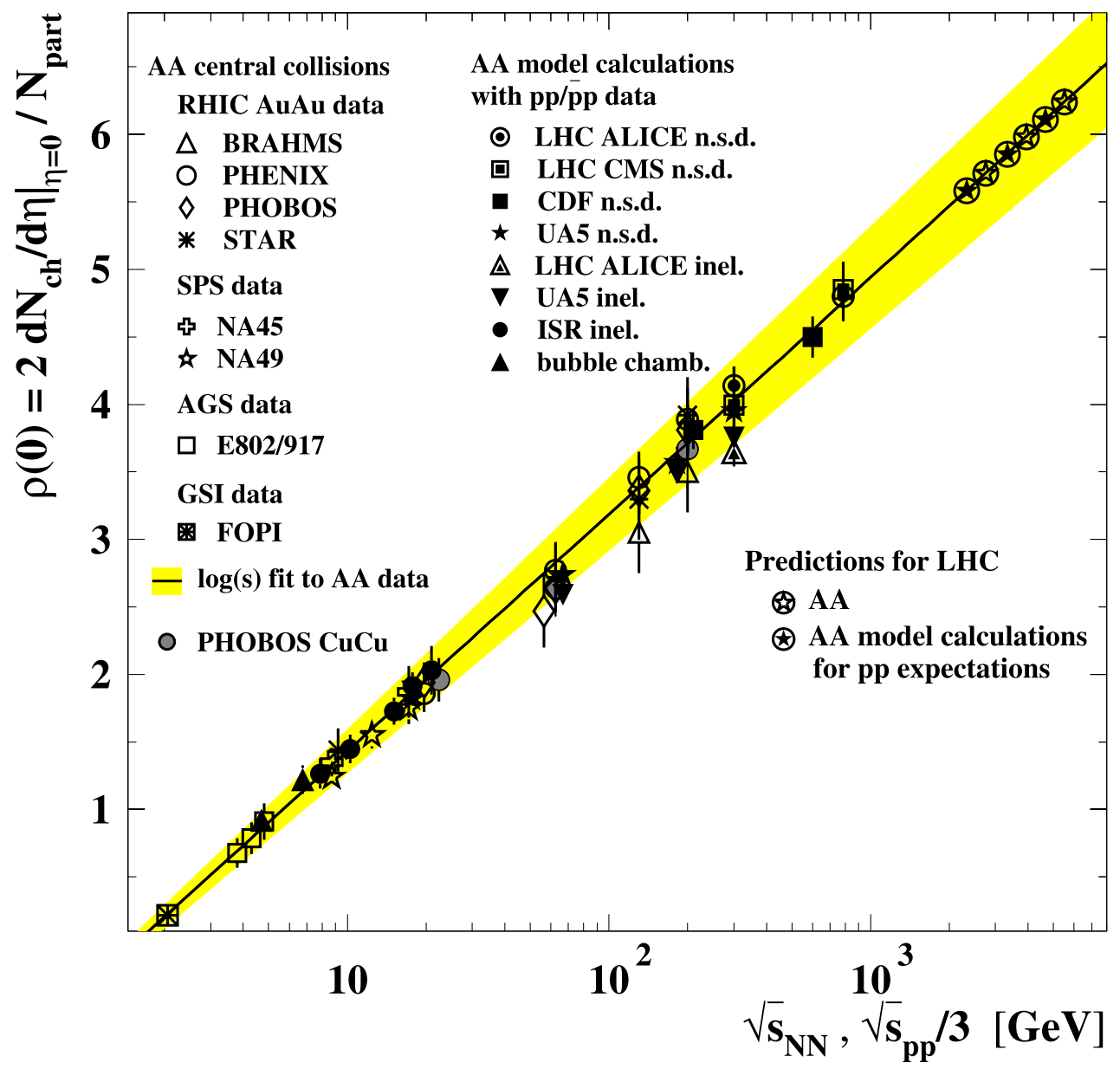

Fig. 1 The pseudorapidity density of charged particles per participant pair at midrapidity as a function of c.m. energy per nucleon, $\sqrt{s_{\mathrm{NN}}}$, measured in central nucleus-nucleus (AA) collisions and calculated from $\mathrm{pp} / \mathrm{p} \mathrm{p}$ data using (2). The AA data are from: the AuAu measurements at RHIC by BRAHMS [31], PHENIX [15, 16], PHOBOS [10, 46-49], and STAR [50-53] experiments; the values recalculated in [15] from the measurements at CERN SPS by CERES/NA45 [44] and NA49 [45] experiments, at Fermilab AGS by E802 and E917 experiments [42, 43], and at GSI by FOPI Collab. [41]; the PHOBOS data on $\mathrm{CuCu}$ collisions from $[48,49,56]$. The solid symbols show the values obtained from (2) using the following data on midrapidity densities measured in non-single diffractive collisions: pp data from

$N_{\mathrm{ch}}$ at $\sqrt{s_{\mathrm{NN}}}$ and the rapidity density $\rho_{\mathrm{pp}}(0)$ and the multiplicity $N_{\mathrm{ch}}^{\mathrm{pp}}$ at $3 \sqrt{s_{\mathrm{NN}}}$. Due to the above, we consider a constituent quark of mass $\frac{1}{3} m_{\mathrm{p}}$ as a participant in $\mathrm{pp} / \overline{\mathrm{p} p}$ collisions, and a proton as an effectively structureless participant in head-on nucleus-nucleus collisions. Then, from (1) one obtains:

$$
\begin{aligned}
& \rho(0)=\rho_{\mathrm{pp}}(0) \frac{2 N_{\mathrm{ch}}}{N_{\mathrm{part}} N_{\mathrm{ch}}^{\mathrm{pp}}} \sqrt{1-\frac{4 \ln 3}{\ln \left(4 m_{\mathrm{p}}^{2} / s_{\mathrm{NN}}\right)}}, \\
& \sqrt{s_{\mathrm{NN}}}=\sqrt{s_{\mathrm{pp}}} / 3 .
\end{aligned}
$$

ALICE [57] and CMS [58] experiments at LHC and from $\overline{\mathrm{p}} \mathrm{p}$ collisions by UA5 Collab. at CERN SPS $[8,59]$ and ISR $\left(\sqrt{s_{\mathrm{pp}}}=53 \mathrm{GeV}\right)$, by CDF Collab. at Fermilab [9]; and in the following inelastic collisions: $\overline{\mathrm{p}} \mathrm{p}$ data by UA5 Collaboration and pp data from the LHC ALICE [57] experiment and from the ISR [60] and bubble chamber [63, 64] experiments, the latter as recalculated in [8]. The solid line shows the linear$\log$ fit, $-0.33+0.38 \ln \left(s_{\mathrm{NN}}\right)$, to the AA data with the parameters and errors obtained using a combination of the data from the RHIC and SPS experiments. The shaded area shows 1- $\sigma$ error band to the fitted parameters. The circled stars show the heavy-ion predictions for LHC AA collisions (open stars) and from the expected LHC pp collisions (solid stars), both calculated from the fit

$\rho(0)$ is thus calculated from the measured values of $\rho_{\mathrm{pp}}(0)$ and the multiplicities measured in both reactions.

Solving (1) for $N_{\mathrm{ch}}$ at given $\rho(0), \rho_{\mathrm{pp}}(0)$ and $N_{\mathrm{ch}}^{\mathrm{pp}}$ one finds:

$\frac{2 N_{\mathrm{ch}}}{N_{\mathrm{part}}}=N_{\mathrm{ch}}^{\mathrm{pp}} \frac{\rho(0)}{\rho_{\mathrm{pp}}(0)} \sqrt{1-\frac{2 \ln 3}{\ln \left(4.5 \sqrt{s_{\mathrm{NN}}} / m_{\mathrm{p}}\right)}}$.

From these one calculates the expected values for nucleusnucleus central collisions, since they are related to $\mathrm{pp} / \overline{\mathrm{p} p}$ interactions through the constituent quarks picture of interactions combined with the Landau energy dissipation hydrodynamics. Note that the different sets of observables used 
in (2) and (3), do not guarantee that both equations describe the data well, even if one of them does.

3. In this section, we consider the available data on the mean multiplicity and midrapidity density measured in nucleus-nucleus collisions.

Figure 1 shows the nuclear data on the pseudorapidity density, $\rho(0)$, per participant pair, measured in head-on nucleus-nucleus collisions (open symbols), as a function of $\sqrt{s_{\mathrm{NN}}}$ in the energy range $\sqrt{s_{\mathrm{NN}}}=2-200 \mathrm{GeV}$. The measurements are from experiments at GSI [41], AGS [42, 43], CERN SPS [44, 45], and RHIC [10, 15, 16, 31, 46-51]. The data clearly satisfies a linear-log relationship. Fitting, using a weighted combination of the data from the RHIC and SPS experiments, we find:

$$
\rho(0)=(-0.327 \pm 0.026)+(0.381 \pm 0.021) \ln \left(s_{\mathrm{NN}}\right) .
$$

Similar observations have been made in [15, 16, 47, 54, 55]. Such a behavior seems [27-29] to be well justified by the participant-driven picture of the formation of the rapidity distribution and the $N_{\text {part }}$ scaling. In addition, as the nuclear data show, the process of bulk production is largely characterized by $N_{\text {part }}$, or soft scaling, rather than by the number of binary collisions, $N_{\text {coll }}$, the latter being attributed to hard scaling [15, 32, 46, 47, 65-67]. Recently, this feature has been confirmed by observing the $N_{\text {part }}$ scaling in the ratio of the charged-particle yields measured at $\sqrt{s_{\mathrm{NN}}}=$ $200 \mathrm{GeV}$ and $62.4 \mathrm{GeV}$, as $\left\langle p_{T}\right\rangle$ increases from $0.25 \mathrm{GeV}$ to $\sim 4 \mathrm{GeV}$ [49]. The increase of the ratio at large $\left\langle p_{T}\right\rangle$ is believed to appear because of the harder spectra at higher $\sqrt{s_{\mathrm{NN}}}$, so as to follow $N_{\text {coll }}$ scaling and not to scale with $N_{\text {part }}$. Interestingly, the $N_{\text {part }}$ scaling is observed to be the same for $\mathrm{AuAu}$ and $\mathrm{CuCu}$ interactions.

Figure 2 shows the $\sqrt{s_{\mathrm{NN}}}$-dependence of the nucleusnucleus data of the mean multiplicity $N_{\text {ch }}$ per participant pair from the most central nucleus-nucleus collisions (solid symbols) in the same $\sqrt{s_{\mathrm{NN}}}$ range as above. The data are taken from the measurements by the PHOBOS experiment at RHIC [4, 5, 32, 46-48], NA49 at CERN SPS [68] and by E895 at AGS [69]. The mean multiplicities are seen to increase as a 2nd-order logarithmic polynomial, and our fit gives:

$$
\begin{aligned}
N_{\text {ch }} /\left(0.5 N_{\text {part }}\right)= & (-0.35 \pm 0.28)+(0.24 \pm 0.16) \ln \left(s_{\mathrm{NN}}\right) \\
& +(0.24 \pm 0.03) \ln ^{2}\left(s_{\mathrm{NN}}\right),
\end{aligned}
$$

which well reproduces the energy-dependence of the measurements. This trend holds even for relatively low-energies, as demonstrated by the recent data by HADES [70] displayed in Fig. 2. Such an $\ln ^{2}\left(s_{\mathrm{NN}}\right)$ behavior is expected [2730] to appear naturally as a combination of the following features: the Landau model Gaussian shape of the pseudorapidity distribution, the logarithmic increase of the midrapidity density with the c.m. energy, and the limiting fragmentation.

4. The midrapidity density and the multiplicity energy dependencies are analyzed in the framework of the model developed here, i.e. applying (2) and (3) to calculate these variables in nucleus-nucleus interactions based on nucleonnucleon data.

Using the $\mathrm{pp} / \overline{\mathrm{p}} \mathrm{p}$ data on $\rho_{\mathrm{pp}}(0)$ from $\mathrm{CERN}^{1}[8,57-60]$ and Fermilab $[9,63,64]$, the data on multiplicities $N_{\mathrm{ch}}^{\mathrm{pp}}$ in pp/p p interactions [59, 60, 64, 73-75], and $N_{\text {ch }}$ from Fig. 2, along with the fact that the transformation factor from rapidity to pseudorapidity does not influence the above ratio, we calculate, according to (2), the $\rho(0)$ at $\sqrt{s_{\mathrm{NN}}}=$ $\sqrt{s_{\mathrm{pp}}} / 3$. These calculations are shown by solid symbols in Fig. 1. One can see that the calculated $\rho(0)$ values are in a very good agreement with the measured $\rho(0)$ 's as well as with the obtained log-fit in the whole measured $\sqrt{s_{\mathrm{NN}}}$ range.

The agreement is more intriguing as one adds the hadronic data at c.m. energies higher than the top RHIC energy, namely the densities for (anti)proton-proton interactions at $\sqrt{s_{\mathrm{pp}}}=1.8 \mathrm{TeV}$ from Tevatron [9] and $\sqrt{s_{\mathrm{pp}}}=$ $2.36 \mathrm{TeV}[57,58]$ from the LHC. In Fig. 1 we compare the calculations using (2) on these TeV-energy data with our linear-log fit. One sees the nucleus-nucleus $\rho(0)$ 's at $\sqrt{s_{\mathrm{NN}}}=600$ and $\sim 800 \mathrm{GeV}$, calculated from the highest energy hadron data, agree well with the AA-fit. This observation justifies the above conclusion from the lowerenergy midrapidity density dependence on the types of participants. Note that (2) shows the relevance of the Landau hydrodynamical energy-dissipation $L$-factor which was required in order to correctly estimate the midrapidity density.

Addressing now (3), we calculate the participant-pairnormalized mean multiplicity $N_{\mathrm{ch}} /\left(0.5 N_{\text {part }}\right)$ for nucleusnucleus interactions from the $\mathrm{pp} / \overline{\mathrm{p} p}$ measurements $[8,9$, 57-60, 63, 64] of $\rho_{\mathrm{pp}}(0)$ and $N_{\mathrm{ch}}^{\mathrm{pp}}, 2$ and the corresponding $\rho(0)$ data from Fig. 1 . The calculated $N_{\text {ch }} /\left(0.5 N_{\text {part }}\right)$ values are shown in Fig. 2 (open symbols) along with the available nuclear data. One can see that the calculated $N_{\mathrm{ch}} /\left(0.5 N_{\text {part }}\right)$ values reproduce well the $\log ^{2}\left(s_{\mathrm{NN}}\right)$ fit obtained here and

\footnotetext{
${ }^{1}$ For the LHC data we exclude some results to avoid any dependence on the experimental cuts and to have consistent data sets. We do not use the $900 \mathrm{GeV}$ data by ATLAS [61] which applies a high $p_{T}$-threshold, and the inelastic (high-energy) data by ALICE [62] where specific selection cut is used. This exclusion does not influence the consideration here.

${ }^{2}$ For the ALICE, CMS and CDF multiplicities the E735 power-law fit $N_{\mathrm{ch}}^{\mathrm{pp}}=3.102 s_{\mathrm{pp}}^{0.178}[72]$ is used.
} 


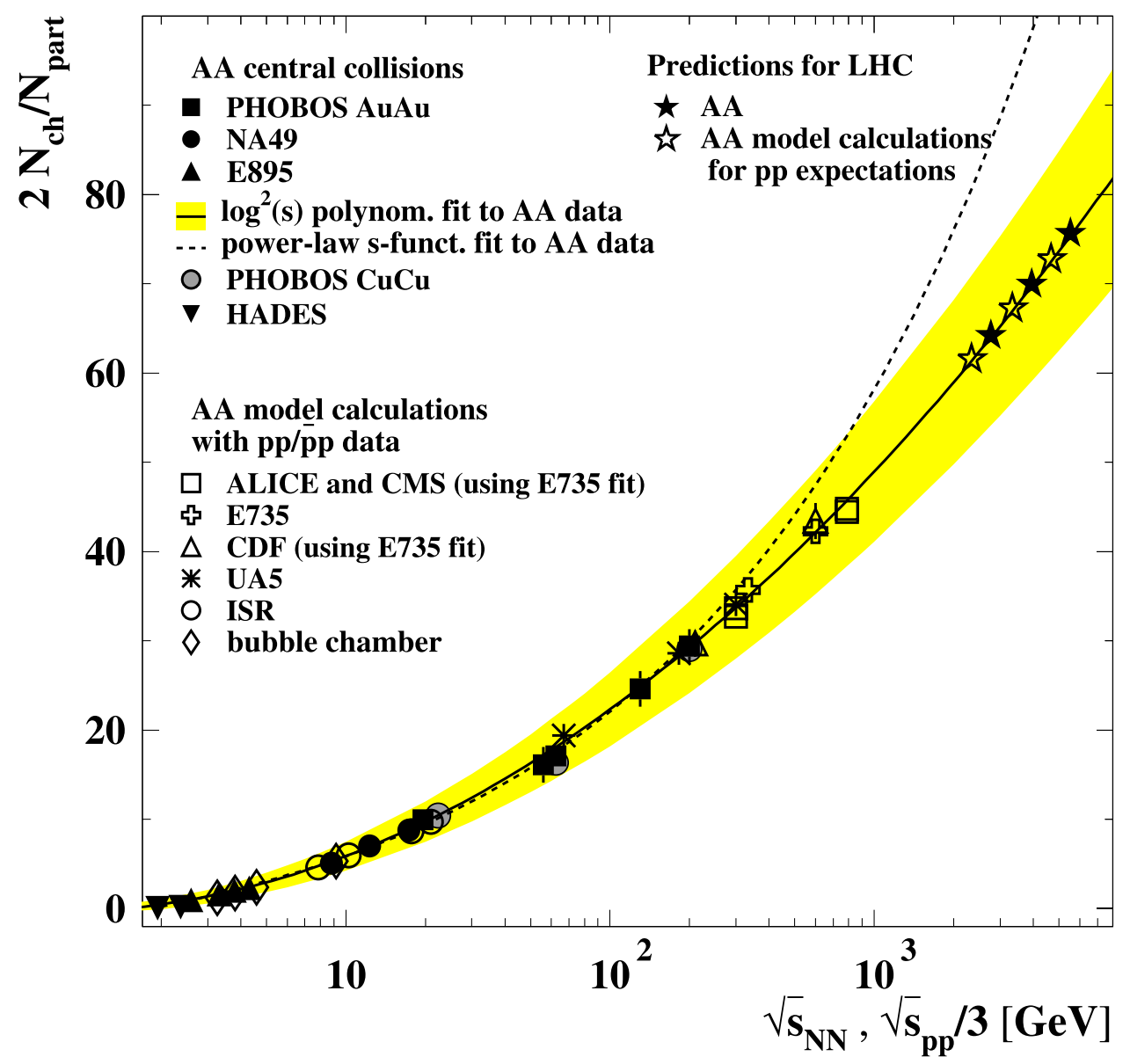

Fig. 2 The charged particle mean multiplicity per participant pair as a function of the nucleon-nucleon c.m. energy, $\sqrt{s_{\mathrm{NN}}}$, measured in the most central nucleus-nucleus (AA) collisions and calculated using $\mathrm{pp} / \overline{\mathrm{p} p}$ data from (3). The solid symbols show the multiplicity values from the AA data as measured by PHOBOS Collab. at RHIC in $\mathrm{AuAu}[4,5,32,46,47]$ and $\mathrm{CuCu}$ [48] collisions, by NA49 Collab. at CERN SPS [68] and by E895 Collab. at AGS [69] (see also [4]). The low-energy HADES measurements at GSI are taken from [70]. The open symbols show the values obtained from (3) for the AA mean multiplicity based on: $\bar{p}$ collisions at FNAL by E735 Collab. [71, 72], at CERN by UA5 Collab. at $\sqrt{s_{\mathrm{pp}}}=546 \mathrm{GeV}$ [59] and $\sqrt{s_{\mathrm{pp}}}=200$ and

follow the nuclear data points for $\sqrt{s_{\mathrm{NN}}}=2 \mathrm{GeV}$ to about $200 \mathrm{GeV}$.

The calculations for the new higher energy data namely those for $\sqrt{s_{\mathrm{NN}}} \gtrsim 200 \mathrm{GeV}$, are again of a special interest. These calculations help to check the proposed description over a larger energy range, the possible fit and result in firmer predictions. Indeed, from Fig. 2, one can see that for $\sqrt{s_{\mathrm{NN}}} \leq 200 \mathrm{GeV}$, it is quite difficult to distinguish between the two fits: the power-law fit $\propto s_{\mathrm{NN}}^{\gamma}$, known to be a preferable fit to the $N_{\mathrm{pp}}^{\text {ch }}$ data $[59,72]$, is almost as good as the $\log ^{2}\left(s_{\mathrm{NN}}\right)$ polynomial approximation. However, it is evident that, after inclusion of the new higher energy data, the $\log ^{2}\left(s_{\mathrm{NN}}\right)$ function is more preferable.
$900 \mathrm{GeV}$ [73]; pp collisions at CERN-ISR [60], and the ALICE, CMS and CDF multiplicities, calculated using the E735 fit, 3.102s $s_{\mathrm{pp}}^{0.178}$ [72], and the data from bubble chamber experiments $[64,74,75]$ the latter having been compiled and analyzed in [76]. The solid line shows the 2nd-order $\log$-polynomial fit $-0.35+0.24 \ln \left(s_{\mathrm{NN}}\right)+0.24 \ln ^{2}\left(s_{\mathrm{NN}}\right)$ to the AA data. The shaded area shows $1-\sigma$ error band to the fitted parameters. The dashed line shows the power-law fit: $-7.32+5.92 s_{\mathrm{NN}}^{0.174}$. The stars give the heavy-ion predictions for the LHC AA collisions (solid symbols) and those expected from LHC pp collisions (open symbols) based on the 2nd-orderlog-polynomial fit

5. From the above, we conclude that using all the world available measurements on the mean multiplicity and the midrapidity density in the data of the nucleon-nucleon and central nucleus-nucleus collisions, a clear interrealtion between the two types of the data is obtained. This can be attributed to the universality of the multiparticle production process over almost three orders of magnitude of $\sqrt{s_{\mathrm{NN}}}$. Under this assumption, predictions for the LHC energies can be made.

Using the fits, shown in Figs. 1 and 2, and solving (1) for the midrapidity density $\rho_{\mathrm{pp}}(0)$ with $N_{\mathrm{ch}}^{\mathrm{pp}}$ from the highenergy fit [72], the expected $\rho_{\mathrm{pp}}(0)$ values for pp collisions at LHC are found to be about 5.8, 6.4, and 6.9 at $\sqrt{s_{\mathrm{pp}}}=$ 7,10 and $14 \mathrm{TeV}$, respectively, within 5\% to $10 \%$ uncertain- 
ties. From the fit to the midrapidity densities $\rho(0)$, shown in Fig. 1, the $\rho(0)$ values, expected for $\mathrm{PbPb}$ collisions at LHC energies $\sqrt{s_{\mathrm{NN}}}$, corresponding to the above $\sqrt{s_{\mathrm{pp}}}$, are found to be about 5.7, 6.0, and 6.2 at $\sqrt{s_{\mathrm{NN}}}=2.76,3.94$ and $5.52 \mathrm{TeV}$, respectively. The $\rho(0)$ predictions are shown in Fig. 1 by circled solid stars for $\mathrm{PbPb}$ interactions and by circled open stars for those from the pp expectations at LHC at $\sqrt{s_{\mathrm{NN}}}=\sqrt{s_{\mathrm{pp}}} / 3$ when calculated according to our model.

Comparing our predictions for $\rho_{\mathrm{pp}}(0)$ to the predictions of other models and Monte Carlo tunes [72, 77], we find that our values are in the range of the midrapidity density values predicted there. The values we find here are also consistent with those from the $\operatorname{CDF} \rho_{\mathrm{pp}}(0)$ fit [9] and from a similar, but higher-energy, CMS fit [58]. The values of $\rho(0)$ we find for LHC heavy-ion collisions are also well in the range of the expectations by different models $[78,79]$. The $\rho(0)$ values obtained at $\sqrt{s_{\mathrm{NN}}} \approx 5.5 \mathrm{TeV}$ is similar to that obtained by PHENIX from the fit to the nuclear data $[15,16]$ and by PHOBOS from their extrapolation of their AuAu data to $\mathrm{PbPb}$ collisions at LHC [27-29].

Using the $\log ^{2} s_{\mathrm{NN}}$ fit to the mean multiplicity shown in Fig. 2, one finds the average multiplicity, $N_{\mathrm{ch}} /\left(0.5 N_{\text {part }}\right)$, in $\mathrm{PbPb}$ collisions to be about 64,70 and 73 at $\sqrt{s_{\mathrm{NN}}}=$ 2.76, 3.94 and $5.52 \mathrm{TeV}$, respectively, with $10 \%$ to $15 \%$ uncertainties. The $N_{\mathrm{ch}}^{\mathrm{pp}}$ in pp collisions at LHC are expected to be about 73,82 and 93 at $\sqrt{s_{\mathrm{pp}}}=7,10$ and $14 \mathrm{TeV}$ within about $10 \%$ uncertainties, and are the same as one finds from the multiplicity high-energy power-law fit [72]. The $N_{\text {ch }} /\left(0.5 N_{\text {part }}\right)$ predictions for $\mathrm{PbPb}$ collisions are shown in Fig. 2 by solid stars, while those expected from the LHC pp collisions at $\sqrt{s_{\mathrm{NN}}}=\sqrt{s_{\mathrm{pp}}} / 3$ in the framework of our model, are shown by open stars there.

The $N_{\mathrm{ch}} /\left(0.5 N_{\mathrm{pp}}\right)$ we obtained are consistent with extrapolations from the present experimental measurements [27-29]. Our prediction at $\sqrt{s_{\mathrm{NN}}} \simeq 5.5 \mathrm{TeV}$ is comparable with the estimate from the pseudorapidity density spectra by PHENIX $[15,16]$. Similar to the $\rho_{\mathrm{pp}}(0)$ values, the $N_{\mathrm{ch}}^{\mathrm{pp}}$ are within the range of the predictions by different models and Monte-Carlo tunes [72, 77].

6. Let us now dwell on some corollaries of the model proposed here and discuss the results in view of other observations.

From our consideration it follows that, at the same $\sqrt{s_{\mathrm{NN}}}$, the mean multiplicities as well as the midrapidity densities, normalized to the number of participants, would give the similar values when measured in central symmetric nucleus-nucleus collisions of different colliding nuclei, they are largely driven by the initial energy deposited by the participants at early stage of collisions. Indeed, as seen from Figs. 1 and 2, this effect has been already observed at SPS energies and now is confirmed by the RHIC measurements at $\sqrt{s_{\mathrm{NN}}}$ of about 50 to $200 \mathrm{GeV}$. The same values for both the observables are obtained [27-29, 54-56, 80] in $\mathrm{Au}-\mathrm{Au}$ and $\mathrm{Cu}-\mathrm{Cu}$ data, as shown in Figs. 1 and 2. Note that this effect has also been observed for the whole pseudorapidity region [81]. Notice also the similarity in the above-discussed $N_{\text {part }}$ dependence in low- $p_{T}$ vs. high $p_{T}$ ranges [49].

An interesting issue to be addressed in the framework of the model is to consider asymmetric collisions, such as nucleon-nucleus ( $\mathrm{pA} / \mathrm{dA})$ ones. In such type of interactions, the bulk variables studied here, being measured at given $\sqrt{s_{\mathrm{NN}}}$ are expected to have the same values as those in $\mathrm{pp} / \overline{\mathrm{p} p}$ collisions at $\sqrt{s_{\mathrm{pp}}} \simeq \sqrt{s_{\mathrm{NN}}}$. Indeed, assuming an incident proton in p-nucleus collisions interacts in the same way it would interact in pp collision, the secondary particles in the reaction are assumed to be created out of the energy deposited by the interaction of a single pair of constituent quarks, one from the proton and another one from a nucleon in the interacting nucleus. This, in its turn, implies that the mean multiplicity and the midrapidity density are expected to be independent of the centrality of nucleus-induced collisions, $N_{\text {part }}$ (within uncertainties due to intranuclear effects, e.g. Fermi motion). These expectation are shown to be well confirmed in the RHIC data on deuteron-gold interactions at $\sqrt{s_{\mathrm{NN}}}=200 \mathrm{GeV}$. Moreover, the effect obtained at RHIC is shown $[5,19,48]$ to be true also for hadron-nucleus collisions at lower $\sqrt{s_{\mathrm{NN}}} \approx 10-20 \mathrm{GeV}$.

In this study the Landau hydrodynamic model is used, leading to a good description of the data from different reactions [5, 18, 23-29, 31-33]. However, this is a $1+1$ model and therefore does not take into account the transverse expansion of the system which can be studied, for example, via the transverse energy, another important bulk observable. Considering the measurements from SPS to RHIC of the transverse energy midrapidity density, one finds that this variable scales with the number of constituent quarks a way similar to that of charged particle and photon midrapidity densities [82]. Furthermore, as measured at RHIC, the ratio of the transverse energy midrapidity density to that of multiplicity density is observed [15] to be independent of the number of participants, and the transverse energy loss is found [83] to be independent of the type of colliding nuclei if the same number of participating nucleons is taken. The observations indicate scalings of a similar nature to those considered here for multiplicities and midrapidity densities. This seems also to reflect the fact that the inclusion of the transverse expansion in the Landau model does not change the scaling of the observables under study [23, 84, 85]. Currently, the model attracts high interest and has undergone a generalization, see e.g. [86-91].

As we have shown, the constituent quark is a key component of a correct description of "soft" particle observables in particle and nuclear collisions from a few GeV up to highest LHC energies, so that constituent quarks have to 
be taken as the interacting particles, see e.g. [92]. This already has support from studies of the multiplicity distributions in heavy-ion-induced interactions [40, 80, 81, 93-95]. The constituent quark picture has been exploited to reasonably model the heavy-ion pseudorapidity and transverse energy data [96]. The elliptic flow characteristics are also observed to scale when the constituent quark frame is taken into account $[97,98]$.

The similarities of $\mathrm{pp} / \mathrm{p} \mathrm{p}$ and $\mathrm{AA}$ interactions observed here for the two basic variables suggest that the system is formed at early stage as a superposition of contributions from the constituent quarks. The particle multiplicities seem then to be derived by the total energy of participants, available in the Lorentz-contracted volume. The importance of the very early stage of collision for soft particle production has been already discussed elsewhere [81, 93, 98, 99]. Due to the proportionality of the multiplicity to the entropy [21, 22], the multiplicity scaling, observed for different variables measured at RHIC, has been suggested to be connected to the total produced entropy $[100,101]$.

Considering this and recalling the above-mentioned similarity in heavy-ion and $\mathrm{e}^{+} \mathrm{e}^{-}$collisions observed, one would expect the same model to be valid in matching the mean multiplicity and the midrapidity values in heavy-ion and $\mathrm{e}^{+} \mathrm{e}^{-}$data. Indeed, as we have shown in [20], both variables follow the same energy dependence within the framework of our picture, as soon as one considers that the structureless electron and positron deposit their total energy into the Lorentz-contracted volume similar to nucleons in central nuclear collisions. From this, the factor $1 / 3$ applied to the $\mathrm{pp} / \overline{\mathrm{p} p}$ energy scale is expected to result in a good match between the $\mathrm{e}^{+} \mathrm{e}^{-}$and $\mathrm{pp} / \overline{\mathrm{p}}$ data on multiplicity and midrapidity densities as shown in [20]. This solves the problem with the energy-scaling factor of $1 / 2$ used in $[4,5]$, where the $\sqrt{s_{\mathrm{pp}}} / 2$ shift is shown to provide a reasonable description of the average multiplicity c.m. energy dependence but not of the midrapidity density when comparing heavyion/ $/ \mathrm{e}^{+} \mathrm{e}^{-}$data to those from $\mathrm{pp} / \overline{\mathrm{p}} \mathrm{p}$ collisions. We recall that the energy-scaling factor $1 / 3$, has already been shown in $[102,103]$ to give good agreement of the pp mean multiplicity data relative to those from $\mathrm{e}^{+} \mathrm{e}^{-}$annihilation, for a review see [1]. It is remarkable that the 3 NLO perturbative QCD [104, 105] fit to $\mathrm{e}^{+} \mathrm{e}^{-}$data [106] describes the $\mathrm{pp} / \overline{\mathrm{p}} \mathrm{p}$ multiplicity data providing the inelasticity is set to $\approx 0.35$ [72], favoring the effective $1 / 3$ c.m. energy in multihadron production in $\mathrm{pp} / \overline{\mathrm{p}} \mathrm{p}$ reactions.

As already mentioned, the average multiplicity is defined mostly by the fraction of the c.m. energy transformed into observed particles, so that, after the energy shift in $\sqrt{s_{\mathrm{pp}}}$ is applied, the pp/p p data reproduce reasonably well the $\mathrm{e}^{+} \mathrm{e}^{-}$ multiplicity data. For the mid-rapidity density, the subsequent system development has to be taken into account and is shown well described by the Landau hydrodynamics picture providing the c.m. energy is scaled according to the contribution of the participants $[20,103]$.

7. In summary, we analyze the average multiplicity and midrapidity density data in $\mathrm{pp} / \mathrm{p} \mathrm{p}$ and in central nuclear interactions as a function of the c.m. energy per nucleon over the whole available range of the interaction c.m. energies, including the highest energy LHC data $\sqrt{s_{\mathrm{pp}}}=2.36 \mathrm{TeV}$ in $\mathrm{pp} / \overline{\mathrm{p}} \mathrm{p}$ collisions, and the highest energy RHIC data at $\sqrt{s_{\mathrm{NN}}}=200 \mathrm{GeV}$. Within the framework of constituent quarks, we develop a model which interrelates these two variables measured in the two types of interactions, assuming one quark of each nucleon participates in $\mathrm{pp} / \overline{\mathrm{p} p}$ collision while all three quarks (i.e. a complete nucleon) participate in a head-on heavy ion collision. We consider these participants to form the initial zone of a collision which then develops in hydrodynamic framework, the Landau relativistic hydrodynamic model in our case. In this approach soft hadron production is determined at the very early stage of the collision. After appropriately taking into account the contributions of the participants, which requires an energyscaling factor of $1 / 3$ in $\mathrm{pp} / \overline{\mathrm{p}} \mathrm{p}$ measurements, the average multiplicity and mid-rapidity density in nucleon-nucleon and nucleus-nucleus interactions are found to have a similar c.m. energy dependence. The midrapidity density is found to obey a linear-log fit on $\sqrt{s_{\mathrm{NN}}}$, while the multiplicity data follows a second-order log-polynomial increase with $\sqrt{s_{\mathrm{NN}}}$. A clear preference of the data on the multiplicity to follow the $\log ^{2}(s)$ behavior is observed compared to the power-law, the two dependencies being indistinguishable up to $\sqrt{s_{\mathrm{NN}}}$ of about $300 \mathrm{GeV}$, or $\sqrt{s_{\mathrm{pp}}} \sim 1 \mathrm{TeV}$. Assuming no changes in the multihadron production processes with increasing energy $\sqrt{s_{\mathrm{pp}}}$ of the LHC to 7,10 and $14 \mathrm{TeV}$, and looking forward to the heavy-ion data at the corresponding $\sqrt{s_{\mathrm{NN}}}$ of $2.76,3.94$ and $5.52 \mathrm{TeV}$, we estimate the multiplicities and midrapidity densities for the forthcoming data, using the obtained energy dependencies.

Acknowledgement We are grateful to David Plane for his help during preparation of the manuscript.

Note added in proof During the publication process of the paper, new data became available from CMS [107] on the pseudorapidity midrapidity density in pp collisions at $7 \mathrm{TeV}$. The value reported is $5.78 \pm 0.01$ (stat) \pm 0.23 (syst), which agrees well with the value of about 5.8 from the prediction made in this paper.

Open Access This article is distributed under the terms of the Creative Commons Attribution Noncommercial License which permits any noncommercial use, distribution, and reproduction in any medium, provided the original author(s) and source are credited. 


\section{References}

1. W. Kittel, E.A. De Wolf, Soft Multihadron Dynamics (World Scientific, Singapore, 2005), p. 652

2. J. Manjavidze, A. Sissakian, Phys. Rep. 346, 1 (2001)

3. I.M. Dremin, J.W. Gary, Phys. Rep. 349, 301 (2001)

4. PHOBOS Collab., B.B. Back et al., Phys. Rev. C 74, 021902 (2006). nucl-ex/0301017

5. PHOBOS Collab., B.B. Back et al., Nucl. Phys. A 757, 28 (2005)

6. A. Białas, B. Bleszyński, W. Czyż, Nucl. Phys. B 111, 461 (1976)

7. A. Białas, W. Czyż, Acta Phys. Pol. B 36, 905 (2005)

8. UA5 Collab., G.J. Alner et al., Z. Phys. C 33, 1 (1986)

9. CDF Collab., F. Abe et al., Phys. Rev. D 41, 2330 (1990)

10. PHOBOS Collab., B.B. Back et al., Phys. Rev. Lett. 85, 3100 (2000)

11. PHENIX Collab., K. Adcox et al., Phys. Rev. Lett. 86, 3500 (2001)

12. PHOBOS Collab., B.B. Back et al., Phys. Rev. Lett. 87, 102303 (2001)

13. PHOBOS Collab., B.B. Back et al., Phys. Rev. Lett. 88, 022302 (2002)

14. PHOBOS Collab., B.B. Back et al., Phys. Rev. C 65, 061901 (2002)

15. PHENIX Collab., S.S. Adler et al., Phys. Rev. C 71, 049901 (2005). Erratum: ibid 71, 034908 (2005)

16. A. Milov (for the PHENIX Collab.), J. Phys. Conf. Ser. 5, 17 (2005)

17. PHENIX Collab., K. Adcox et al., Nucl. Phys. A 757, 184 (2005)

18. BRAHMS Collab., I. Arsene et al., Nucl. Phys. A 757, 1 (2005)

19. B.B. Back et al., Phys. Rev. C 72, 031901 (2005)

20. E.K.G. Sarkisyan, A.S. Sakharov, AIP Conf. Proc. 828, 35 (2005). hep-ph/0410324

21. L.D. Landau, Izv. Akad. Nauk: Ser. Fiz. 17, 51 (1953). English translation: Collected Papers of L.D. Landau, ed. by D. TerHaarp (Pergamon, Oxford, 1965), p. 569. Reprinted in: QuarkGluon Plasma: Theoretical Foundations, ed. by J. Kapusta, B. Müller, J. Rafelski (Elsevier, Amsterdam, 2003), p. 283

22. S.Z. Belenkij, L.D. Landau, Phys. Uspekhi 56, 309 (1955). English translation: Collected Papers of L.D. Landau, p. 665, and Nuovo Cimento, Suppl. 3, 15 (1956)

23. E.L. Feinberg, in Relativistic Heavy Ion Physics, ed. by L.P. Csernai, D.D. Strottman. Int. Rev. Nucl. Phys., vol. 6 (World Scientific, Singapore, 1991), p 341, and refs. therein

24. P.A. Carruthers, M. Duong-van, Phys. Rev. D 8, 859 (1973)

25. P. Steinberg, J. Phys. Conf. Ser. 9, 280 (2005)

26. P. Steinberg, Nucl. Phys. A 752, 423c (2005)

27. W. Busza, Acta Phys. Pol. B 35, 2873 (2004)

28. W. Busza, J. Phys. G 35, 044040 (2008)

29. W. Busza, Nucl. Phys. A 830, 35c (2009)

30. M.-A. Sanchis-Lozano, Int. J. Mod. Phys. A 24, 4529 (2009)

31. BRAHMS Collab., I.G. Bearden et al., Phys. Rev. Lett. 88, 202301 (2002)

32. B.B. Back et al., Phys. Rev. Lett. 91, 052303 (2003)

33. BRAHMS Collab., I.G. Bearden et al., Phys. Rev. Lett. 94, 162301 (2005)

34. J. Benecke, T.T. Chou, C.N. Yang, E. Yen, Phys. Rev. 188, 2159 (1969)

35. J.D. Bjorken, Phys. Rev. D 27, 140 (1983)

36. For reviews, see: V.V. Anisovich, N.M. Kobrinsky, J. Nyiri, Yu.M. Shabelsky, Quark Model and High Energy Collisions (World Scientific, Singapore, 2004).

37. J. Nyiri, Int. J. Mod. Phys. A 18, 2403 (2003)

38. M. Basile et al., Nuovo Cimento A 66, 129 (1981)

39. M. Basile et al., Nuovo Cimento A 73, 329 (1983)

40. S. Eremin, S. Voloshin, Phys. Rev. C 67, 064905 (2003)
41. Estimated in [15] from: FOPI Collab., W. Reisdorf et al., Nucl. Phys. A 612, 493 (1997)

42. E802 Collab., L. Ahle et al., Phys. Rev. C 59, 2173 (1999)

43. E917 Collab., B.B. Back et al., Phys. Rev. Lett. 86, 1970 (2001)

44. F. Ceretto (for the CERES/NA45 Collab.), Nucl. Phys. A 638, 467c (1998)

45. F. Siklér (for the NA49 Collab.), Nucl. Phys. A 661, 45c (1999)

46. PHOBOS Collab., B.B. Back et al., Phys. Rev. C 70, 021902(R) (2004)

47. PHOBOS Collab., B.B. Back et al., Phys. Rev. C 74, 021901(R) (2006)

48. B. Alver et al., Phys. Rev. Lett. 102, 142301 (2009)

49. PHOBOS Collab., B. Alver et al., Phys. Rev. C 80, 011901(R) (2009)

50. STAR Collab., C. Adler et al., Phys. Rev. Lett. 87, 112303 (2001)

51. STAR Collab., B.I. Abelev et al., Phys. Rev. C 79, 034909 (2009)

52. STAR Collab., B.I. Abelev et al., Phys. Rev. C 81, 024911 (2010)

53. L. Kumar (for the STAR Collab.), J. Phys. G 36, 064066 (2009)

54. R. Nouicer, AIP Conf. Proc. 828, 11 (2005)

55. R. Nouicer, AIP Conf. Proc. 842, 86 (2006)

56. R. Nouicer, arXiv:0901.0910 [nucl-ex]

57. ALICE Collab., K. Aamondt et al., Eur. Phys. J. C 68, 89 (2010)

58. CMS Collab., V. Khachatryan et al., JHEP 02, 041 (2010)

59. UA5 Collab., G.J. Alner et al., Phys. Rep. 154, 247 (1987)

60. W. Thomé et al., Nucl. Phys. B 129, 365 (1977)

61. ATLAS Collab., G. Aad et al., Phys. Lett. B 688, 21 (2010)

62. ALICE Collab., K. Aamondt et al., Eur. Phys. J. C 68, 345 (2010)

63. J. Whitmore et al., Phys. Rep. 10C, 273 (1974)

64. W.M. Morse et al., Phys. Rev. D 15, 66 (1977)

65. A. Iordanova (for the PHOBOS Collab.), Czech. J. Phys. 56, A39 (2006)

66. B.B. Back (for the PHOBOS Collab.), nucl-ex/0604017

67. G.S.F. Stephans (for the PHOBOS Collab.), 0705.3859 [nucl-ex]

68. NA49 Collab., S.V. Afanasiev et al., Phys. Rev. C 66, 054902 (2002)

69. E895 Collab.: J.L. Klay, PhD thesis (U.C. Davis, 2001), see [4]

70. HADES Collab., G. Agakishev et al., Eur. Phys. J. A 40, 45 (2009)

71. C.S. Lindsey, Nucl. Phys. A 544, 343c (1992)

72. J.F. Grosse-Oetringhaus, K. Reygers, J. Phys. G 37, 083001 (2010)

73. UA5 Collab., R.E. Ansorge et al., Z. Phys. C 43, 357 (1989)

74. V.V. Ammosov et al., Phys. Lett. B 42, 519 (1972)

75. C. Bromberg et al., Phys. Rev. Lett. 31, 254 (1974)

76. E. De Wolf, J.J. Dumont, F. Verbeure, Nucl. Phys. B 87, 325 (1975)

77. A. Moraes, C. Buttar, I. Dawson, Eur. Phys. J. C 50, 435 (2007)

78. N. Armesto, in Quark-Gluon Plasma 4, ed. by R.C. Hwa, X.-N. Wang (World Scientific, Singapore, 2010), p. 375. arXiv:0903.1330 [hep-ph]

79. M. Mitrovski et al., Phys. Rev. C 79, 044901 (2009)

80. R. Nouicer, Eur. Phys. J. C 49, 281 (2007)

81. A. Białas, A. Bzdak, Phys. Rev. C 77, 034908 (2008)

82. P.K. Netrakanti, B. Mohanty, Phys. Rev. C 70, 027901 (2004)

83. B. Alver et al., Phys. Rev. Lett. 96, 212301 (2006)

84. G.A. Milekhin, Sov. Phys. JETP 35, 829 (1959)

85. E.V. Shuryak, Sov. J. Nucl. Phys. 20, 295 (1974)

86. A. Bialas, R.A. Janik, R. Peschanski, Phys. Rev. C 76, 054901 (2007)

87. L.M. Satarov, I.N. Mishustin, A.V. Merdeev, H. Stöcker, Phys. Rev. C 75, 024903 (2007)

88. L.M. Satarov, I.N. Mishustin, A.V. Merdeev, H. Stöcker, Phys. Atom. Nucl. 70, 1773 (2007)

89. Ch.-Y. Wong, Phys. Rev. C 78, 054902 (2008). arXiv: 0809.0517 [nucl-th]

90. T. Mizoguchi, H. Miyazawa, M. Biyajima, Eur. Phys. J. A 40, 99 (2009) 
91. M. Zétényi, L.P. Csernai, Phys. Rev. C 81, 044908 (2010)

92. S. Bondarenko, E. Levin, Eur. Phys. J. C 51, 659 (2007)

93. A. Bialas, J. Phys. G 35, 044053 (2008)

94. F.-H. Liu, Nucl. Phys. A 801, 154 (2008)

95. F.-H. Liu, Nucl. Phys. A 810, 159 (2008)

96. B. De, S. Bhattacharyya, Phys. Rev. C 71, 024903 (2005)

97. PHOBOS Collab., B. Alver et al., Phys. Rev. Lett. 98, 24302 (2007)

98. P. Steinberg, Nucl. Phys. A 827, 128c (2009)

99. W. Ochs, V.A. Khoze, M.G. Ryskin, Eur. Phys. J. C 68, 141 (2010)
100. H. Caines, Eur. Phys. J. C 49, 297 (2007)

101. G. Torrieri, Phys. Rev. C 76, 024903 (2007)

102. P.V. Chliapnikov, V.A. Uvarov, Phys. Lett. B 251, 192 (1990)

103. T.F. Hoang, Z. Phys. C 62, 481 (1994)

104. I.M. Dremin, J.W. Gary, Phys. Lett. B 459, 341 (1999)

105. A. Capella et al., Phys. Rev. D 61, 074009 (2000). For review see: I.M. Dremin, J.W. Gary [3]

106. ALEPH Collab., A. Heister et al., Eur. Phys. J. C 35, 457 (2004)

107. CMS Collab., V. Khachatryan et al., Phys. Rev. Lett. 105, 022002 (2010) 